\title{
A CLINICAL PROFILE OF TUBERCULOSIS IN CKD PATIENTS AND THE RESPONSE TO MODIFIED ATT
}

\author{
Anusuya Meganathan', S. Anu²
}

${ }^{1}$ Associate Professor, Department of General Medicine, Government Stanley Medical College and Hospital, The Tamilnadu Dr. MGR Medical University, Chennai, Tamilnadu, India.

2Postgraduate Student, Department of General Medicine, Government Stanley Medical College and Hospital, The Tamilnadu Dr. MGR Medical University, Chennai, Tamilnadu, India.

\begin{tabular}{l}
\hline ABSTRACT \\
BACKGROUND \\
Tuberculosis (TB) is one of the major causes of mortality across the world. Patients with CKD are at high risk of developing TB due \\
to the immunosuppressive effects of uraemia. There is limited information on the magnitude of problem of TB in patients with \\
CKD.
\end{tabular}

Aim: The aim of the study was to find out the clinical profile and response to treatment of TB in CKD patients.

\section{MATERIALS AND METHODS}

This is a descriptive study carried out on 55 cases of CKD. The patients were grouped into various stages of CKD based on GFR calculated using the MDRD equation. The microbiological, histopathological, radiological and newer diagnostic investigations were noted for each patient with TB. Also, the duration of ATT taken, and any incidence of adverse drug reaction were observed.

\section{RESULTS}

In our study subjects, the mean age was 40 yrs., of which $75 \%$ constituted males and $25 \%$ females. Majority the patients were of post-renal transplant and stage V (31\% each). Pulmonary TB contributed $64 \%$ cases (Majority in Stage IV and V) and extrapulmonary TB 36\% (especially in post-renal transplant subjects). The mean duration of ATT was 7 months with 28 cases of treatment completed, 23 cured, 1 failed and 3 defaulters. Only 1.8\% patients developed adverse drug event in the form of peripheral neuropathy, due to INH, despite taking pyridoxine supplements.

\section{CONCLUSION}

Incidence of Pulmonary TB is more common in post-transplant subjects, whereas extrapulmonary TB (Lymph node) is more common in CKD subjects. A high index of suspicion should be maintained by ensuring early screening for risk factors like smoking, alcoholism and diabetes mellitus as they predispose CKD patients to tuberculosis. GeneXpert assay is a novel and reliable diagnostic modality for TB, especially in CKD subjects for whom many of the conventional methods may be negative.

\section{KEY WORDS}

Chronic Kidney Disease, Dialysis, Haemodialysis, Post-Renal Transplant, Pulmonary Tuberculosis, Anti-Tuberculosis Treatment. HOW TO CITE THIS ARTICLE: Meganathan A, Anu S. A clinical profile of tuberculosis in CKD patients and the response to modified ATT. J. Evolution Med. Dent. Sci. 2018;7(38):4143-4147, DOI: 10.14260/jemds/2018/927

\section{BACKGROUND}

Tuberculosis (TB) is a major public health problem, especially in the developing countries like Asia and Africa. There is a global surge in the prevalence of tuberculosis due to immigration, poverty, HIV infection and rise of drug-resistant strains. ${ }^{1,2,3}$ The incidence of Chronic Kidney Disease is increasing due to the changing lifestyle and habits. Patients with chronic renal failure are particularly at risk for acquiring $\mathrm{TB}$, because of impaired cellular immunity. 4

This is true for all patients with chronic kidney disease (CKD), 30 times, ${ }^{5}$ higher prevalence of TB in patients with $\mathrm{CRF}$. The incidence of $\mathrm{TB}$ in Indian patients receiving maintenance haemodialysis has been reported to be 3.7 to $13.3 \%{ }^{6}$

'Financial or Other Competing Interest': None.

Submission 01-08-2018, Peer Review 01-09-2018,

Acceptance 06-09-2018, Published 17-09-2018.

Corresponding Author:

Dr. Anusuya Meganathan,

\#351, Konnur High Road,

C-47, Sunnyvale Apartments,

Ayanavaram, Chennai-600023,

Tamilnadu, India.

E-mail: anusuyarubi@gmail.com

DOI: $10.14260 /$ jemds $/ 2018 / 927$
Incidence of TB in renal transplant recipients has ranged from 1 to 4\% in Northern Europe, 0.5 - 1\% in North America and nearly 5 to $10 \%$ in India. ${ }^{7}$ Studies showed that $46 \%$ of the renal failure patients present adverse reactions to the anti-tuberculosis drugs used, a percentage even higher in dialysed patients with the same pathology.

Chronic Kidney Disease (CKD) is defined as kidney damage or an estimated Glomerular Filtration Rate (eGFR) below $60 \mathrm{~mL} / \mathrm{min} / 1.73 \mathrm{~m} 2$ persisting for 3 months or more irrespective of the cause. The Kidney Disease Outcomes Quality Initiative (KDOQI) guidelines classified CKD into 5 stages. (Addition of the suffix $\mathrm{T}$ for renal allografts and suffix D to identify CKD stage 5 patients on dialysis).

\section{Staging of CKD-K/DOQI 2002, Modified by NICE 2008}

1. Stage 1 CKD: Normal GFR: Some evidence of kidney damage reflected by microalbuminuria, proteinuria, haematuria as well as radiologic/ histologic changes.

2. Stage 2 CKD: eGFR $60-89 \mathrm{~mL} / \mathrm{min} / 1.73$ sq. $\mathrm{m}$

3. Stage 3a CKD: eGFR $59-45 \mathrm{~mL} / \mathrm{min}$

4. Stage 3b CKD: eGFR $44-30 \mathrm{~mL} / \mathrm{min}$

5. Stage 4 CKD: eGFR $29-15 \mathrm{~mL} / \mathrm{min}$

6. Stage 5 CKD/ESRD: eGFR $<15 \mathrm{~mL} / \mathrm{min}$ 
An acid fast bacilli-positive smear, positive culture of M. tuberculosis and typical histopathologic findings makes the diagnosis of TB. Appropriate materials should be obtained for culture and sensitivity testing.

In $40-100 \%$ of the cases, the diagnosis of TB is hampered by the occurrence of a negative Purified Protein Derivative (PPD) (Mantoux) skin test. A high index of suspicion is required coupled with a need for invasive procedures, including liver, bone, lymph node and peritoneal biopsies should be the dictum due to the frequent extrapulmonary presentation and non-specific symptomatology.

\section{Aims}

This study was done to find out the clinical profile of tuberculosis in chronic kidney disease patients and their response to anti-tubercular treatment including adverse.

\section{The Objectives are-}

1. To study the clinical profile and associated risk factors of TB in CKD patients.

2. To study the prevalence of TB in CKD patients on/ not on dialysis/ post-transplant recipients.

3. To study the pattern of involvement (pulmonary/ extrapulmonary) of TB in the same category of patients.

4. To study the response to modified ATT in those patientscured/failure/treatment completed/died/ developed drug toxicity.

\section{MATERIALS AND METHODS}

\section{Study Area}

This study was carried out in the Department of Nephrology at Government Stanley Medical College and Hospital, Chennai, Tamilnadu.

\section{Study Population}

CKD patients diagnosed and admitted for treatment and renal transplant recipients diagnosed with tuberculosis.

\section{Study Period}

October 2016 to May 2017.

\section{Sample Size}

A minimum of 55 CKD patients were recruited for the study through convenience sampling method.

\section{Inclusion Criteria}

- $\quad$ CKD patients stage III - V on/ not on dialysis.

- Renal transplant recipients.

- Diagnosed to have tuberculosis by microbiology or histopathology or radiological suspicion.

\section{Exclusion Criteria}

- Patients not on regular follow-up.

- Poor drug compliance.

\section{Study Design}

It is a descriptive study.

\section{Study Outcome Variables Study Tools}

1. Detailed history using standard questionnaire. Age, symptomatology, non-CKD risk factors like smoking, alcoholism, previous history of pulmonary TB, presence of diabetes mellitus, other infections like HCV and in case of post-transplant patients, use of induction therapy, any graft dysfunction etc. was noted.

2. Clinical examination was done and patients were grouped into various stages of CKD based on eGFR calculated using the MDRD equation. The diagnostic investigation was noted for each patient with TBmicrobiologic/ histopathologic/ radiologic/ newer modalities. The duration of anti-tuberculous therapy taken, adverse drug reactions if any etc. was noted. In relation to definitions, patients with pulmonary tuberculosis with bacteriologically confirmed TB at the beginning of treatment who is smear or culture negative in the last month of treatment and on at least one previous testing was considered as cured, patients with diagnosed tuberculosis who completed treatment without evidence of failure but there is no record to show that sputum smear or culture results in the last month of treatment and on at least one previous occasion were negative, either because the tests were not done or results not available was considered as treatment completed and patients with tuberculosis whose sputum smear or culture is positive at the end of 5 months of ATT or later during treatment were considered as treatment failure.

\section{Statistical Analysis}

The collected data were analysed with SPSS statistical software 16.0 Version. Descriptive statistics was calculated and used. Percentages were used for categorical variables and the mean and standard deviation was used for continuous variables. Chi-square test and student's t-test was used to test statistical significance, which was fixed at $<0.05$.

\section{RESULTS}

\begin{tabular}{|c|c|}
\hline Study Variable & Frequency (\%) \\
\hline \multicolumn{2}{|c|}{ Age } \\
\hline$\leq 25$ years & $4(7.3)$ \\
\hline $26-35$ years & $19(34.5)$ \\
\hline $36-45$ years & $16(29.1)$ \\
\hline $46-55$ years & $9(16.4)$ \\
\hline$>55$ years & $7(12.7)$ \\
\hline \multicolumn{2}{|c|}{ Gender } \\
\hline Female & $14(25.5)$ \\
\hline Male & $41(74.5)$ \\
\hline \multicolumn{2}{|c|}{ Stage of CKD } \\
\hline III & $2(3.6)$ \\
\hline IV & $12(21.8)$ \\
\hline V & 17 (30.9) \\
\hline $\mathrm{Vd}$ & $7(12.7)$ \\
\hline $\mathrm{Vt}$ & $17(30.9)$ \\
\hline \multicolumn{2}{|c|}{ Smoking } \\
\hline No & $41(74.5)$ \\
\hline Yes & $14(25.5)$ \\
\hline \multicolumn{2}{|c|}{ Alcoholism } \\
\hline No & $50(90.9)$ \\
\hline Yes & $5(9.1)$ \\
\hline \multicolumn{2}{|c|}{ Old PT } \\
\hline No & $52(94.5)$ \\
\hline Yes & $3(5.5)$ \\
\hline \multicolumn{2}{|c|}{ Type 2 DM } \\
\hline No & $49(89.1)$ \\
\hline
\end{tabular}




\begin{tabular}{|c|c|}
\hline Yes & $6(10.9)$ \\
\hline \multicolumn{2}{|c|}{ New Onset Type 2 DM } \\
\hline No & $53(96.4)$ \\
\hline Yes & $2(3.6)$ \\
\hline \multicolumn{2}{|c|}{ Graft Dysfunction } \\
\hline No & $52(94.5)$ \\
\hline Yes & $3(5.5)$ \\
\hline \multicolumn{2}{|c|}{ HCV Positive } \\
\hline No & $53(96.4)$ \\
\hline Yes & $2(3.6)$ \\
\hline \multicolumn{2}{|c|}{ Site of TB } \\
\hline $\mathrm{PTB}$ & $35(63.6)$ \\
\hline EP TB & $20(36.4)$ \\
\hline \multicolumn{2}{|c|}{ Response to Treatment } \\
\hline Cured & $18(32.7)$ \\
\hline Defaulter & $3(5.5)$ \\
\hline Pending & $5(9.1)$ \\
\hline Treatment Completed & $28(50.9)$ \\
\hline Treatment Failure & $1(1.8)$ \\
\hline \multicolumn{2}{|c|}{ ADR } \\
\hline Nil & $54(98.2)$ \\
\hline To INH & $1(1.8)$ \\
\hline \multicolumn{2}{|c|}{ Induction Therapy } \\
\hline Nil & $46(83.6)$ \\
\hline Yes & $9(16.4)$ \\
\hline \multicolumn{2}{|c|}{ TB Mortality } \\
\hline No & $54(98.2)$ \\
\hline Yes & $1(1.8)$ \\
\hline $\begin{array}{r}\text { Table 1. Distribution of D } \\
\text { of Stud }\end{array}$ & $\begin{array}{l}\text { ind Baseline Details } \\
\text { ts }\end{array}$ \\
\hline
\end{tabular}

Almost 820 patients with CKD inclusive of transplant recipients are screened for tuberculosis to get population of 57 patients with CKD and tuberculosis, of which only 55 patients inducted in our study as per inclusion and exclusion criteria. With this data, prevalence is estimated to be $6.9 \%$ in our study.

As depicted in Table 1 the age ranged between $17-63$ years, the percentage of sample between age class intervals of upto 25 years, 26 - 35 years, 36 - 45 years, 46 - 55 years and $>55$ years were $7.3,34.5,29.1,16.4,12.7$ respectively. Majority of the sample population lies between 26 - 35 years. Mean age of the sample was 39.71 with a standard deviation of 11.13. The mean duration of anti-tuberculosis therapy was 6.78 months and the duration ranged between 4 - 12 months with a standard deviation of 1.83 . The mean duration on dialysis (for patients coming under category CKD V D) was 7 months with a range of $3-12$ months and a standard deviation of 2.89. In this study, $74.5 \%$ were male patients and $25.5 \%$ were females.

Around $30.9 \%$ of the sample belonged to CKD stage Vt and Vd each respectively, $21.8 \%$ to stage IV, $12.7 \%$ to stage Vd and $3.6 \%$ to stage III. It was also found that $25.5 \%$ of the patients with TB were smokers and $9.1 \%$ of the patients with TB were alcoholics. To be exact, 5.5\% of the TB patients had history of prior pulmonary tuberculosis. Incidence of diabetes mellitus was $10.9 \%$ in our study and $3.6 \%$ of the population had new-onset diabetes mellitus after transplant. With respect to graft dysfunction, $5.5 \%$ of the sample had evidence of it and $3.6 \%$ of the population were HCV positive. Of all the patients, $64 \%$ had pulmonary TB and $36 \%$ had extrapulmonary TB.
In our study participants $28 \%$ of the patients completed treatment, $18 \%$ were declared cured, $5 \%$ were on ongoing treatment, $3 \%$ defaulted and $1 \%$ were treatment failed cases. Only $1.8 \%$ of patients ( 1 patient) developed adverse drug event, which was to INH in the form of peripheral neuropathy despite taking pyridoxine supplements. It was observed that $16.4 \%$ of patients had received induction therapy with monoclonal antibodies / anti-thymocyte globulin and 1.8\% (1 patient) died of tuberculosis.

\begin{tabular}{|c|c|}
\hline Diagnostic Investigations & Frequency (\%) \\
\hline Ascitic Fluid AFB+, Bactec MTB + & $1(1.8)$ \\
\hline Aspirate GeneXpert+, Granuloma+ & $2(3.6)$ \\
\hline BAL MTB + & $4(7.3)$ \\
\hline CSF GeneXpert MTB+ & $1(1.8)$ \\
\hline CT Chest & $2(3.6)$ \\
\hline Exudative Pleural Fluid, High ADA & $11(20.0)$ \\
\hline FNA LN- Granuloma & $6(3.6)$ \\
\hline High Protein CSF, GeneXpert MTB+ & $1(1.8)$ \\
\hline LN Excision Biopsy Positive & $3(5.5)$ \\
\hline MRI Spine & $4(7.3)$ \\
\hline Sputum AFB+ & $13(23.6)$ \\
\hline Sputum GeneXpert & $2(3.6)$ \\
\hline Synovial Biopsy & $5(7.3)$ \\
\hline \multicolumn{2}{|c|}{$\begin{array}{c}\text { Table 2. Distribution of Diagnostic Investigations } \\
\text { of Study Participants }\end{array}$} \\
\hline
\end{tabular}

Table 2 depicts the relative percentages of various diagnostic modalities: $23.6 \%$ - sputum positivity for acid fast bacilli, 20\% - exudative pleural fluid with high ADA, 7.3\% Bronchoalveolar lavage MTB detected, $7.3 \%$ - MRI spine, $5.5 \%$ - lymph node excision biopsy positive, $3.6 \%$ each for sputum, ascitic fluid GeneXpert positivity, lymph node fine needle aspiration positivity, positive findings on CT chest and $1.8 \%$ for CSF GeneXpert positivity.

\begin{tabular}{|c|c|c|}
\hline $\begin{array}{c}\text { Stage of } \\
\text { CKD }\end{array}$ & Pulmonary (n=35) & Extrapulmonary (n=20) \\
\hline III & $1(5.0)$ & $1(2.9)$ \\
\hline IV & $1(5.0)$ & $11(31.4)$ \\
\hline V & $6(30.0)$ & $11(31.4)$ \\
\hline Vd & $0(0.0)$ & $7(20.0)$ \\
\hline Vt & $12(60.0)$ & $5(14.3)$ \\
\hline \multicolumn{2}{|c|}{ Table 3. Association of Stage of CKD with Site of TB in } \\
Study Participants
\end{tabular}

The comparison between stages of CKD and TB types (Table 3) shows that there is a high statistical significance $(p=0.002)$. The relative percentages of pulmonary and extrapulmonary TB was done stage wise and it was found that in case of pulmonary TB maximum percentage of cases were of stage IV and V. In case of extrapulmonary, maximum percentage of cases were of CKD stage $V$ post-transplant status. $38.2 \%$ of the cases were pulmonary which topped in number followed by pleural (20\%), then skeletal and synovial (18.2\%), then lymph node $(14.5 \%)$ followed by CNS $(3.6 \%)$, peritoneal, miliary and disseminated (1.8\% each). Regarding the pattern of presentation cough was the major complaint, which comprised of $45.5 \%$ followed by weight loss (30.9\%), 
fever $(23.6 \%)$ and haemoptysis (16.4\%). While observing pattern of chest imaging, consolidation (23.6\%) outnumbered cavitation (18.2\%) and lower lobe involvement (3.6\%).

\begin{tabular}{|c|c|c|}
\hline $\begin{array}{c}\text { Pattern of } \\
\text { Involvement }\end{array}$ & CKD Vd & CKD Vt \\
\hline \multicolumn{3}{|c|}{ Pulmonary } \\
\hline Yes & $0(0.0)$ & $12(70.6)$ \\
\hline No & $7(100)$ & $5(29.4)$ \\
\hline \multicolumn{3}{|c|}{ Pleural } \\
\hline Yes & $12(28.6)$ & $0(0.0)$ \\
\hline No & $5(71.4)$ & $17(100)$ \\
\hline \multicolumn{3}{|c|}{ Abdominal and Peritoneal } \\
\hline Yes & $2(28.6)$ & $0(0.0)$ \\
\hline No & $5(71.4)$ & $17(100)$ \\
\hline \multicolumn{3}{|c|}{ Skeletal and Synovial } \\
\hline No & $0(0.0)$ & $4(23.5)$ \\
\hline Yes & $7(100)$ & $13(73.5)$ \\
\hline \multicolumn{3}{|c|}{ Alcoholism } \\
\hline No & $0(0.0)$ & $4(23.5)$ \\
\hline Yes & $7(100)$ & $13(73.5)$ \\
\hline \multicolumn{3}{|c|}{ Lymph Node } \\
\hline No & $3(42.9)$ & $0(0.0)$ \\
\hline Yes & $4(57.1)$ & $17(100)$ \\
\hline \multicolumn{3}{|c|}{ CNS } \\
\hline No & $0(0.0)$ & $1(5.9)$ \\
\hline Yes & $7(100)$ & $16(94.1)$ \\
\hline \multicolumn{3}{|c|}{$\begin{array}{l}\text { Table 4. Association of Dialysis and Post-Transplant CK } \\
\text { Status with Pattern of Involvement of TB in Study } \\
\text { Participants }\end{array}$} \\
\hline
\end{tabular}

\begin{tabular}{|c|c|c|}
\hline Risk Factors & CKD Vd & CKD Vt \\
\hline \multicolumn{3}{|c|}{ Hypoalbuminaemia } \\
\hline Yes & $2(28.6)$ & $4(23.5)$ \\
\hline No & $5(71.4)$ & $13(76.5)$ \\
\hline Les & $4(57.1)$ & $7(47.2)$ \\
\hline No & $3(42.9)$ & $10(58.8)$ \\
\hline \multicolumn{3}{|c|}{ Diabetes Mellitus } \\
\hline Yes & $3(57.1)$ & $6(64.7)$ \\
\hline No & $4(42.9)$ & $11(35.3)$ \\
\hline \multicolumn{3}{|c|}{ Skeletal and Synovial } \\
\hline $\begin{array}{r}\text { Table 5. Association of Dialysis and Post-Transplant CKD } \\
\text { Status with Risk Factors of TB in Study Participants }\end{array}$ \\
\hline
\end{tabular}

The comparison between CKD VD and CKD VT in respect to pattern of involvement of TB within showed statistically significant results in pulmonary $(\mathrm{p}=0.005)$ and lymph node ( $p=0.012)$, while it was non-significant in pleural, abdominal/peritoneal, skeletal/synovial and CNS (Table 4). On analysis of the risk factors in comparison between CKD VD and CKD VT, it was observed that hypoalbuminaemia, low BMI and Diabetes Mellitus were non-significant (Table 5). Among post-transplant patients, majority (70.6\%) had pulmonary TB in contrast to other groups of CKD under study who had extrapulmonary (78.9\%) as the major type of TB.

\section{DISCUSSION}

Tuberculosis (TB) is one of the major causes of mortality across the world. Because of the immunosuppressive effect of uraemia, patients with Chronic Kidney Disease (CKD) are at high risk of developing TB. In this study, there were a total of 55 patients with CKD diagnosed as tuberculosis by various means- clinical, histopathology, biochemical, microbiologic, radiologic or a combination of factors. The mean age of the study subjects was 40 years. $75 \%$ of the subjects were males and $25 \%$ females. Majority of the subjects were of post renal transplant and stage $\mathrm{V}$ (31\% each).

Among the risk factors for tuberculosis, smoking contributed $26 \%$ followed by diabetes mellitus (11\%). Majority of smokers among the group developed pulmonary tuberculosis highlighting the association between smoking and pulmonary tuberculosis. $16.4 \%$ of the subjects (i.e. $53 \%$ of the post-transplant subjects) received induction therapy with monoclonal antibodies/ antithymocyte globulin. This signifies the fact that immunosuppression caused by these agents can have a predisposition to the development of tuberculosis. $5.5 \%$ of the subjects developed graft dysfunction requiring treatment with plasmapheresis/IVIG/ Rituximab/steroids also pointing to the role of predisposition caused by these agents to develop tuberculosis.

In this study prevalence of CKD with tuberculosis was estimated to be $6.9 \%$ in our study, which is on par with other Indian studies. ${ }^{7}$ Pulmonary tuberculosis contributed $64 \%$ of cases (majority in stages IV and V CKD) and extrapulmonary $36 \%$ (maximum number in post renal transplant subjects). Of the latter group, pleural TB topped in number accounting to $20 \%$ of cases followed by skeletal TB, lymph node and abdominal TB and disseminated TB being least in number. On comparing the CKD patients on dialysis vs post-transplant, the observation was that pulmonary tuberculosis was significantly increased in the latter group. Lymph node tuberculosis was significantly increased in the former group compared to the latter. There was not much statistically significant association between the risk factors like hypoalbuminaemia, low BMI and diabetes mellitus between the two groups.

On comparing the post-transplant subjects with the remaining CKD patients under study, it was found that pulmonary tuberculosis was the predominant type in the former group (71\%), whereas extrapulmonary (79\%) was the predominant type in the latter group. The diagnostic investigation was sputum positive for acid fast bacilli in majority of patients followed by exudative pleural fluid with elevated ADA. The role of bronchoalveolar lavage; however, cannot be underestimated since sputum negative cases could be diagnosed with accuracy using it (in 7.3\% of cases among the subjects, BAL AFB positivity was the diagnostic investigation).

Regarding GeneXpert assay, it contributed to $9 \%$ of the diagnostic investigatory modalities of all among the study subjects. When the conventional diagnostic modalities show inconclusive results, it stands useful and reliable. Imaging modalities too at times help in diagnosing as shown by the fact that $3.6 \%$ of cases were diagnosed at PT based on CT chest and showed improvement clinically and radiologically, though other modes of diagnoses came out as negative.

The mean duration of antituberculous therapy was 7 months with 28 cases of treatment completed ones, 18 cured, 5 ongoing, 1 failed and 3 defaulters. The high number of former two categories indicates the efficacy of the present system of directly observed treatment short course being followed in our part of the world.

Among the patients on dialysis the mean duration of dialysis was 7 months, only $1.8 \%$ of patients (1 patient) developed adverse drug event which was to INH in the form 
of peripheral neuropathy despite taking pyridoxine supplements. Only 1 patient had death due to tuberculosis, a case of CKD IV, SHT, sputum positive pulmonary TB with respiratory failure.

\section{The above Results on Comparison to other International and Indian Statistics and Studies-}

- The prevalence of TB in post-transplant cases between 2014 - 2016 in our hospital as per the study (since all the post-transplant CKD under study underwent transplantation between this time period) is $17 / 120 \mathrm{x}$ $100=14.17 \%$, which is very much comparable with that of most of the Indian studies (5-14\%) 21 like Sakhuja et al as already discussed above.

- The risk factors like smoking, alcoholism, prior TB, T2DM like previous studies remain relevant in present study too. Factors like graft dysfunction, NODAT and HCV positivity too were seen in common in some cases of tuberculosis in post-transplant patients, whether they have any role as a contributing factor to tuberculosis needs further studies to explain.

- GeneXpert assay stands as a novel and significant investigatory modality for TB added onto the conventional ones already being described in prior studies.

- In contrast to prior studies where typical investigatory tests like sputum AFB positivity, pleural fluid analysis were inconclusive, in the present study they still remain the most significant, probably since the study group contained fairly high number of post-transplant subjects and also since pleuropulmonary was the major type of TB among them.

- Extrapulmonary TB is the major type in CKD stages III, IV, Vd (lymph node TB being the major type) and pulmonary being the major type in CKD Vt in par with studies. 8,9

- CNS, skeletal, abdominal, pleural TB does not have statistically significant difference in percentage when compared between post-transplant CKD and subjects on dialysis in contrast to Tony et al study. ${ }^{9}$

- The response to treatment has been good enough showing high cure and treatment completed rates in contrast to prior studies ${ }^{10}$ indicating efficacy of DOTS.

\section{CONCLUSION}

In our study, we can safely conclude that Tuberculosis is a common infection among Chronic Kidney Disease patients, due to the immunosuppressed state. Hence, a high index of suspicion should be maintained with ways of early screening to be followed based on clinical grounds. Risk factors namely smoking, alcoholism, diabetes mellitus predisposes CKD patients to tuberculosis in addition to the immunosuppressed state of CKD. GeneXpert assay is a novel, reliable diagnostic modality for tuberculosis, especially in CKD subjects for whom many of the conventional methods may be negative and bronchoalveolar lavage increases the detection rates of AFB. Pulmonary TB is more common in post-transplant patients, whereas extrapulmonary (lymph node) tuberculosis is more common in both CKD-HD and CKD-NHD groups. The tuberculosis cure rates are reasonably good with timely follow-up, monitoring by renal and pulmonary physicians.

\section{REFERENCES}

[1] Dunlap N, Bailey WC. A catastrophe is brewing feditorian. Chest 1993;103:332-4.

[2] Vas SI. Renaissance of tuberculosis in the 1990s: lessons for the nephrologist. Perit Dial Int 1994;14(3):209-14

[3] Bhatti N, Law MR, Morris JK, et al. Increasing incidence of tuberculosis in England and Wales: a study of the likely causes. Br Med J 1995;310(6985):967-9.

[4] Descamps-Latscha B. Infection and immunity in endstage renal disease. In: Henrich WL, edr. Principles and practice of dialysis. Baltimore, USA: Williams and Wilkins 1994: p. 209-24.

[5] Yuan FH, GuanLX, Zhao SJ. Patients with renal disease are at increased risk of tuberculosis (TB). This is true for all patients with chronic kidney disease (CKD), 30 times higher prevalence of TB in patients with CRF. Renal Fal 2005;27:149-53.

[6] Narula AS. The incidence of TB in Indian patients receiving maintenance hemodialysis has been reported to be 3.7 to 13.3 percent. Indian J Nephrol 1991;1:67.

[7] McWhinney N, Khan O, Williams G. Tuberculosis in patients undergoing maintenance haemodialysis and renal transplantation. Br Surg 1981;68(6):408-11.

[8] Sakhuja V, Jha V, Varma PP, et al. The high incidence of tuberculosis among renal transplant recipients in India. Transplantation 1996;61(2):211-5.

[9] Walls T, Shingadia D. The epidemiology of tuberculosis in Europe. Arch Dis Child 2007;92(8):726-9.

[10] Cox HS, Morrow M, Deutschmann PW. Long term efficacy of DOTS regimens for tuberculosis: systematic review. BMJ 2008;336(7642):484-7. 